\title{
Unveiling the afterlife of galaxies with ultraviolet data
}

\author{
Ariel Werle ${ }^{1,2}$ (D) \\ ${ }^{1}$ INAF - Osservatorio Astronomico di Padova, Vicolo dell'Osservatorio 5, 35122 Padova, Italy \\ ${ }^{2}$ Instituto de Astronomia, Geofísica e Ciências Atmosféricas, Universidade de São Paulo, \\ R. do Matão 1226, 05508-090 São Paulo, Brazil \\ email: ariel.werle@inaf.it
}

\begin{abstract}
Recent works have shown that early-type galaxies (ETGs) are much more complex than early studies suggested. We present early results from a combined analysis of optical spectra and ultraviolet photometry for a sample of 3453 red sequence galaxies in at $z<0.1$ that are classified as elliptical by Galaxy Zoo. By measuring the Gini index of the star-formation histories derived by STARLIGHT, we investigate the complexity of the mixture of stellar populations required to describe ETGs in our sample. When fitting only optical spectra, STARLIGHT assigns more or less the same mixture of stellar populations to all ETGs, while the addition of UV data unveils a bimodallity in the star-formation histories of these galaxies. We find evidence for stellar populations younger than 1 Gyr in 17 per cent of our sample, indicating that some galaxies do not stay permanently quenched after reaching the red sequence.
\end{abstract}

Keywords. galaxies: evolution, galaxies: elliptical and lenticular, $\mathrm{cD}$, ultraviolet: galaxies

\section{Introduction}

Studies of the stellar populations of early-type galaxies (ETGs) based on their optical stellar continuum are unable to capture some nuances in their star-formation histories. Fortunately, this limitation can be surpassed with the addition of ultraviolet data, which allows the detection of even small amounts of young stellar populations, and also provides information on old hot stars (e.g. horizontal branch, extreme horizontal branch and post-asymptotic giant branch).

Here we present early results of the simultaneous analysis of optical spectra from the Sloan Digital Sky Survey (SDSS, York et al. 2000) Data Realease 7 (Abazajian et al. 2009) and photometry from the Galaxy Evolution Explorer (GALEX, Martin et al. 2005) in the NUV and FUV bands for a sample of 3453 galaxies with $N U V-r>5$ at $z<0.1$ that are classified as elliptical by Galaxy Zoo. For this, we use a new version of the STARLIGHT spectral synthesis code (Cid Fernandes et al. 2005), wich allows the combined analysis of spectroscopic and photometric data (Werle et al. 2019). Our spectral fitting is based on an updated version of the Bruzual \& Charlot (2003) stellar population models (Charlot \& Bruzual in prep, private communication).

\section{Analysis}

STARLIGHT decomposes galaxy spectra into a non-parametric combination of stellar population models. Here we use the fraction of stellar mass attributed by STARLIGHT to each stellar population model $(\mu)$ to compute mass-weighted mean stellar ages $\left(\langle\log t\rangle_{M}\right)$ and also the Gini index of $\mu$ for ETGs in our sample. The lower the Gini index, the more STARLIGHT has to mix different stellar populations in order to match GALEX and SDSS

(C) The Author(s), 2021. Published by Cambridge University Press on behalf of International Astronomical Union. This is an Open Access article, distributed under the terms of the Creative Commons Attribution licence (http://creativecommons.org/licenses/by/4.0/), which permits unrestricted re-use, distribution, and reproduction in any medium, provided the original work is properly cited. 


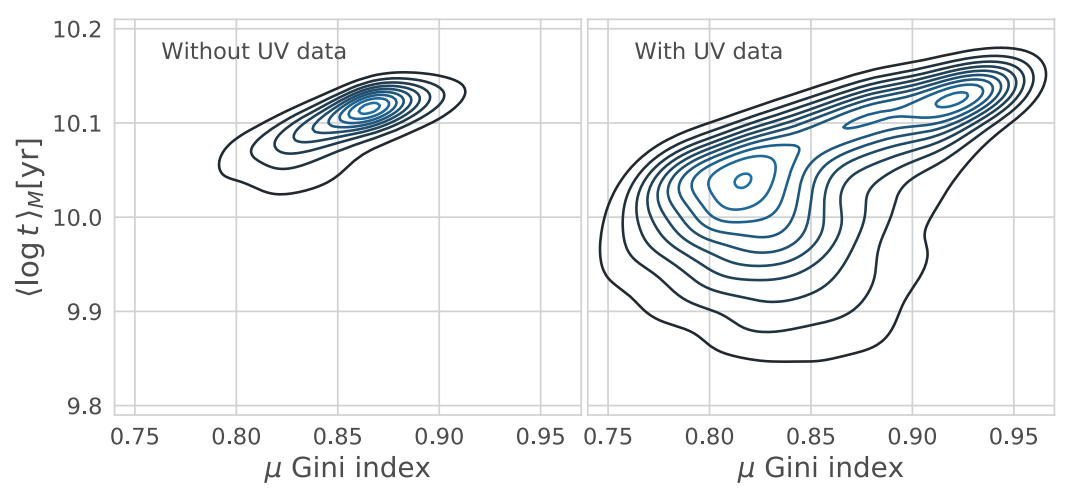

Figure 1. Gini index of the stellar populations against mass-weighted mean stellar ages for STARLIGHT fits of optical spectra (left) and for fits taking into account the combination of optical spectra and ultraviolet photometry (right).

data of a given galaxy. If the Gini index is equal to 1 , the galaxy is fitted by one single stellar population model.

In Fig. 1, we plot the Gini index of $\mu$ against $\langle\log t\rangle_{M}$ for two different sets of STARLIGHT fits. When fitting only SDSS spectra (left panel), our sample covers a small range of values in both Gini index and age. With the addition of GALEX data (right panel), a bimodality appears, allowing us to distinguish between two sub-populations. While some galaxies are described solely by very old stellar populations (large Gini index and old age), others had more extended periods of star-formation (lower Gini index and younger age). Stellar populations younger than $1 \mathrm{Gyr}$ are required to fit the GALEX photometry of about 17 per cent of galaxies in our sample.

\section{Final remarks}

The combined analysis of SDSS spectra and GALEX photometry reveals a bimodality in the star-formation histories of ETGs. Our results indicate that some galaxies do not stay permanently quenched after reaching the red sequence, as has been found by a number of studies in recent years. Instead, some early-type galaxies may undergo "rejuvenation events" triggered by minor mergers or the accretion of cold gas from the intergalactic medium. These and others results from our spectral synthesis will be explored in more detail in Werle et al., in prep.

\section{References}

Abazajian, K. N., et al. 2009, ApJS, 182, 543

Bruzual, G. \& Charlot S. 2003, MNRAS, 344, 1000

Cid Fernandes, R., Mateus, A., Sodré, L., et al. 2005, MNRAS, 358, 363

Martin, D. C., et al. 2005, ApJ, 619, L1

Werle, A., Cid Fernandes, R., Vale Asari, N., et al. 2019, MNRAS, 483, 2382

York, D. G., et al. 2000, AJ, 120, 1579 